\title{
A fine balance
}

The molecular mechanisms underlying the link between ageing and neurodegeneration are unclear. Now, a new study suggests that the expression levels of the miR-34 microRNA (miRNA) and the E74A transcription factor connect these processes in Drosophila melanogaster.

miRNA pathways are increasingly recognized to have vital roles in

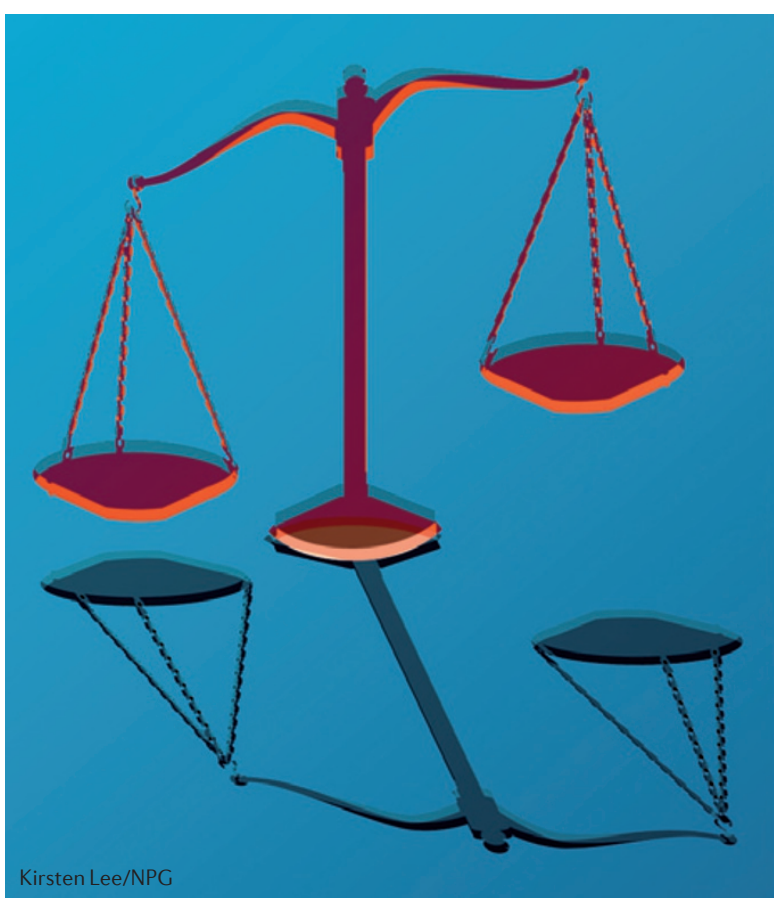

maintaining the health of the nervous system during adulthood in various species. Here, the authors showed that flies bearing a hypomorphic mutation in loquacious, a key gene in miRNA processing, exhibited late-onset changes in brain morphology and had shortened lifespans. Interestingly, miRNA microarray analysis of RNA from normal flies at different ages revealed that among miRNAs expressed in the adult brain, miR-34 was selectively upregulated as the flies aged.

To elucidate the specific function of miR-34, the authors generated miR-34-knockout flies. These mutants had no obvious developmental defects, but they did show a dramatic decline in adult viability after 30 days, thus highlighting the adult-onset nature of miR-34 function. Moreover, the mutant flies showed brain vacuolization, akin to that seen in much older wild-type flies. Interestingly, upregulation of miR-34 expression enhanced the median lifespan of wild-type flies. Together, these findings suggest that miR-34 regulates age-associated phenotypes in flies through protecting against neurodegeneration.

The authors showed that miR-34 binds to the $3^{\prime}$ untranslated region of the Ecdysone-induced protein $74 E F$ (Eip74EF) gene, which is a component of steroid hormone signalling pathways that have been implicated in regulating lifespan. This gene encodes two isoforms, and the authors found that miR-34 specifically downregulates the expression of the E74A isoform during adulthood. Interestingly, abnormal upregulation of E74A expression in flies decreased lifespan and caused late-onset brain defects, indicating that miR-34 may function to protect against E74A-mediated neurodegenerative effects. The authors found, however, that upregulation of miR-34 also had E74A-independent neuroprotective effects in a fly model of polyglutamine disease, revealing that miR-34 is likely to confer neuroprotection through multiple pathways.

miR-34 is evolutionarily conserved from flies to mammals. Thus, the authors suggest that further study of this miRNA may shed light on the mysteries behind ageing and susceptibility to developing neurodegenerative disease.

Man Tsuey Tse

ORIGINAL RESEARCH PAPER Liu, N. et al. The microRNA miR-34 modulates ageing and neurodegeneration in Drosophila. Nature $\mathbf{4 8 2}$, 519-523 (2012) 